\title{
Seasonal variation in Google search interest for melasma
}

\author{
Suraj Muddasani ${ }^{1}$, Sean Z. Wu², Alan B. Fleischer Jr. ${ }^{2}$ \\ ${ }^{1}$ College of Medicine, University of Cincinnati, 3230 Eden Ave, Cincinnati, OH 45267, USA, ${ }^{2}$ Department of Dermatology, \\ University of Cincinnati, 3230 Eden Ave., Cincinnati OH 45267, USA
}

Corresponding author: Suraj Muddasani, E-mail: muddassr@mail.uc.edu

Sir,

Melasma is a common chronic acquired disorder of hyperpigmentation found worldwide. The relationship between sun exposure and melasma exacerbation is commonly reported, as is the use of broad-spectrum sunscreens in treating melasma [1]. However, the seasonal variations in the prevalence or incidence of melasma are not well established, having only been examined in a single-center study in Nepal [2]. In the absence of large-scale clinical epidemiological studies, we used Google search data to conduct an ecological study of seasonal patterns of public interest in melasma.

Google Trends was queried for the search term "melasma" from January 1, 2011 to December 31, 2019 among users in the United States (US). Google Trends outputs search interest data that is normalized by time and geographic region. The single maximal search interest value in each query is set as "100", and all other points are scaled proportionally. There are mechanisms in place to detect and filter irregular activity. Searches made very infrequently, duplicate searches from the same person over a short period and searches with special characters are filtered out [3]. Data from January 1, 2020 and after was excluded to minimize the effect of the novel coronavirus-19 pandemic on search results. The data was acquired on May 13, 2020 [4].

Cosinor analysis, which models seasonal patterns using a sinusoidal equation, was performed on the dataset [5]. Cosinor analyses yield two $\mathrm{p}$ values, a sine and cosine $\mathrm{P}$ value. If either $\mathrm{p}$-value is less than the established value of significance, the model detects significant seasonality.
Using the Bonferroni correction to address the multiple comparison problem, the level of significance was established at $p<0.025$ [5] In addition to providing a seasonal peak and nadir, the model also provides an amplitude which is half the extent of predictable variation in a cycle. Cosinor analysis was performed in R version 3.6.3 with "season" package [6,7].

A time series of monthly normalized search interest for melasma in the US showed clear seasonal variations that cycled annually (Figs. la and lb). Cosinor analysis showed that this seasonality in the US was statistically significant $(\sin p<0.001, \cos p<0.001)$, with an amplitude of 22.1, a peak in June, and a nadir in December (Fig. 1c).

This exploratory study shows that in the US, a country with a primarily temperate climate, Google search interest for melasma peaked in the summertime. This pattern of seasonality of public interest in melasma is unsurprising, given prior clinical reports of UV as a trigger for melasma and a short theoretical latency period between exposure and disease exacerbation [1]. However, we cannot exclude another confounding variable driving these seasonal differences. A limitation of the cosinor model is that it assumes a sinusoidal pattern, which must be symmetric and may be too restrictive in certain cases [5]. Furthermore, how well seasonal variations in public interest, as measured by Google search interest, mirror differences in incidence requires further epidemiological or clinical studies.

\section{Consent}

The examination of the patient was conducted according to the Declaration of Helsinki principles. 


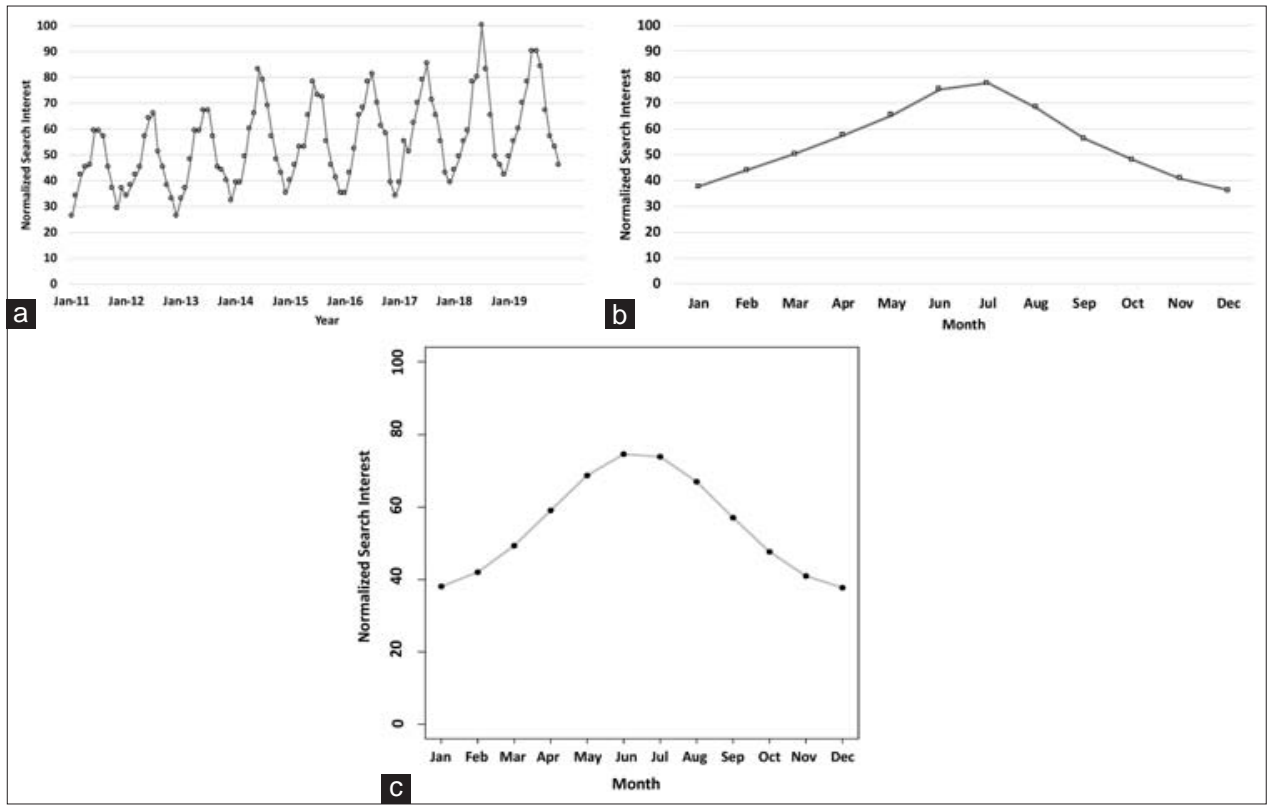

Figure 1: Graphical representations of normalized search interest, A. normalized search interest time series, B. mean normalized search interest for each month of the year, C. cosinor models of normalized search interest, Jan=January, Feb=February, Mar=March, Apr=April, May=May, Jun=June, Jul=July, Aug=August, Sep=September, Oct=October, Nov=November, Dec=December.

\section{REFERENCES}

1. Sheth VM, Pandya AG. Melasma: a comprehensive update: part II. J Am Acad Dermatol. 2011;65:699-714.

2. Jha AK, Gurung D. Seasonal variation of skin diseases in Nepal: a hospital based annual study of out-patient visits. Nepal Med Coll J. 2006;8:266-8.

3. FAQ about Google Trends data. Google. https://support.google. $\mathrm{com} /$ trends/answer/4365533?hl=en. Accessed May 13, 2020.

4. Google Trends. https://trends.google.com/trends/. Accessed May 13, 2020.

5. Barnett AG. DA. Analysing Seasonal Health Data. Berlin, Heidelberg: Springer; 2010.

6. Barnett AG BP. Season: seasonal analysis of health data. R package version 0.3-5. https://cran.r-project.org/package=season $/$. Accessed April 18,2020.
7. R: a language and environment for statistical computing. $\mathrm{R}$ Foundation for Statistical Computing. https://www.R-project.org/. Accessed April 18, 2020.

Copyright by Suraj Muddasani, et al. This is an open-access article distributed under the terms of the Creative Commons Attribution License, which permits unrestricted use, distribution, and reproduction in any medium, provided the original author and source are credited.

Source of Support: Nil, Conflict of Interest: Dr. Fleischer is a consultant for Boerhringer-Ingelheim, Dermavant, Incyte, Qurient, SCM Lifescience and Syneos. He is an investigator for Galderma, Menlo and Trevi. He has no other potential conflicts including Honoraria, Speakers bureau, Stock ownership or options, Expert testimony, Grants, Patents filed, received, pending, or in preparation, Royalties, or Donation of medical equipment. 